\title{
Implementation of PCCD-OFDM-ASK Robust Data Transmission over GSM Speech Channel
}

\author{
Zdenko MEZGEC, Amor CHOWDHURY, Bojan KOTNIK \\ Ultra d.o.o., Research Center \\ Gosposvetska ul. 84, SI-2000 Maribor, Slovenia \\ e-mail:zdenko.mezgec@ultra.si,bojan.kotnik@ultra.si \\ Rajko SVEČKO \\ Faculty of Electrical Engineering and Computer Science, University of Maribor \\ Smetanova ul. 17, SI-2000 Maribor, Slovenia \\ e-mail:rajko.cvecko@uni-mb.si
}

Received: April 2008; accepted: August 2008

\begin{abstract}
This paper presents a novel data modulation scheme PCCD-OFDM-ASK: the phase continuous context dependent orthogonal frequency division multiplexing amplitude shift keying. The proposed modulation is successfully applied in the mobile payment system. It is used to transmit the digital data over the speech channel of the mobile communication system GSM, as well as CDMA. The main key points of the proposed modulation schemes are: precise signal synchronization between the modulator and demodulator, signal energy independent operation, on line adaptation of frequency characteristics of the transmission channel, and controlled frequency bandwidth thus enabling non-overlapped full duplex communication over the GSM's voice channel.
\end{abstract}

Keywords: digital modulations, OFDM, ASK, GSM, speech coders.

\section{Introduction}

A mobile phone is an indispensable device in everyday life. Its basic function is to transmit human speech. The Mobile telecommunication technology is experiencing an unprecedented worldwide success story. We often come across mobile trading, mobile business, and mobile payments, and these functions use different data transmission systems. One of them is data transmission over the speech channel of different mobile communication systems such as GSM (Global System for Mobile Communications), CDMA (Code division Multiple Access) and UMTS (Universal Mobile Telecommunication System). This type of transmission is already incorporated into the presented mobile transaction system and has already obtained two international patents (Ultra M-Pay Patent 1 and 2, 2002). The basis of this mobile transaction system is voice-coded data transmission between the processing centre and the terminal (Fig. 1).

The mobile transaction POS (Point of sale) terminal works similarly like a card POS terminals. The system is generally designed for two types of terminals (Fig. 2); the mPOS (mobile POS) and aPOS(automatic POS). 


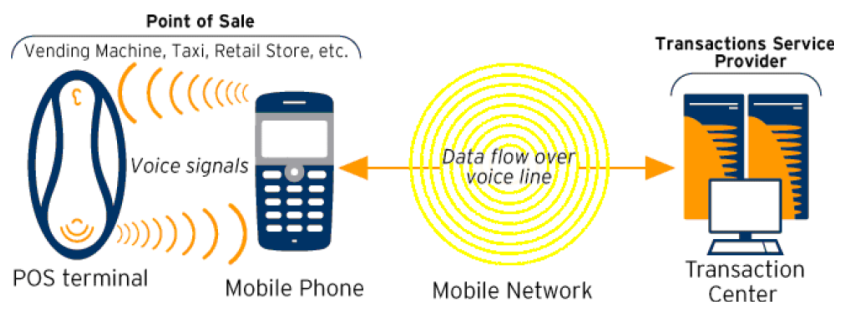

Fig. 1. The basis of mobile transaction system.

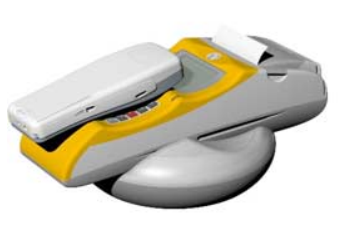

mPOS T4000

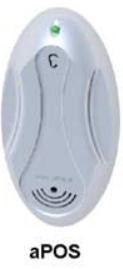

apos

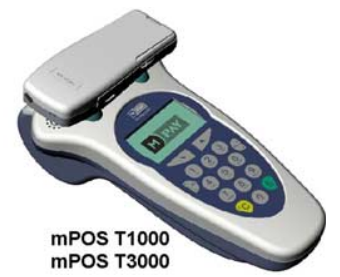

Fig. 2. mPOS and aPOS terminals.

The mPOS terminal is used in restaurants, shops, taxis, hyper markets, etc., while aPOS terminal is connected to other devices like vending machines, car, wash machines, electric counters, etc. Both terminals hardware equipment are much alike. There are two main differences between them. The aPOS terminal does not have the hardware equipment necessary for the interaction with human. The mPOS terminal does not have the hardware equipment necessary for the interaction with different vending machines.

\subsection{Why Using Data Transmission over the GSM's Speech Channel}

There are various technologies such as NFC (Near Field Communication), GPRS (General Packer Radio Service), UMTS, HSDPA (High-Speed Downlink Protocol Access), Wi-Fi (Wireless Fidelity), SMS (Short Message Service) and others that can be used for high-speed data transmission as well as for providing mobile transactions services (micro payments, macro payments, bill payments, prepaid electronic top-up and bonus loyalty programs) (Chow et al., 1991). But in opposition to the above mentioned data transmission techniques, there are some important advantages of the proposed mobile payment system incorporating data transmission principle over the voice channel of the GSM system:

- Passive terminal communication: all data is transmitted and received through speech channel of user mobile phone.

- Independent of the mobile phone standard, because all phones have their primary function of transmitting and receiving the voice.

- Online authorization for each transaction.

- No communication costs for merchant, because there is no other connectivity besides transmission over the mobile phone speech channel. 
- Identification of the user through the MSISDN (Mobile Subscriber ISDN number).

- Only small amount of the transaction data must be transmitted. Therefore, there is no need for high data transmission rates.

- No physical connection between the user's GSM terminal and the POS terminal is needed. The user's GSM terminal and the POS terminal are coupled only via voice only (microphone-speaker remote coupling). Therefore, any type of the user's GSM terminal is supported to perform mobile payment transaction using the proposed data transmission system.

- The above mentioned facts enable the usage of the proposed mobile payment system worldwide, almost regardless of the quality and novelty of the existing GSM infrastructure. Therefore this system makes sense in taking further business and development of the presented technology.

The aim of this paper is to present improvements and optimization of data transmission between the terminal and processing center. The object is not to achieve maximum possible transmission bit rate, but most of all making a good compromise between adequate bit rate, high reliability, and robustness of the real data transmission system. For better understanding we must present next two important facts that were considered in presented research. One of them is that transactions must be as fast as possible, because online authorizations may not exceed time over around 10 seconds. This time can vary a lot from one authorization institution to other. Second information is data size that needs to be transmitted. Size of all data for both transmit and receive is around 250 bytes but final size is off course related to type of techniques that we use for robust transmission, such as BEC (backward error correction) and FEC (forward error correction). Those two techniques can add more than $100 \%$ overhead if the chosen modulation technique does not give enough robustness and because of that we need to use better FEC.

\subsection{Overall Organization of the Paper}

The organization of the paper is as follows. Section 2 provides short description of the GSM mobile telecommunication system and the properties of the speech coding techniques applied in the GSM. A brief overview of known digital modulation algorithms that have been used as research origin is presented in Section 3. Section 4 is the core part of this paper. It presents the algorithmic details of the proposed novel modulation technique PCCD-OFDM-ASK. The comparative evaluation and verification results from off-line experiments and on-line real environment application are collected in Section 5. Section 6 concludes the paper with our final ascertainment.

\section{Speech Coding in GSM}

GSM is the pan-European cellular mobile standard (Scourias, 1995). Three speech coding algorithms are part of this standard (GSM 06.10 Full Rate (FR) Vocoder, 1996; GSM 06.60 Enhanced Full Rate (EFR) Vocoder, 1997; GSM 06.20 Half Rate (HR) Vocoder, 
1998). The purpose of these coders is to compress the speech signal before its transmission, reducing the number of bits needed in its digital representation, while keeping an acceptable perceived quality of the decoded output speech signal. As GSM transcoding (the process of coding and decoding) modifies the speech signal, it is likely to have a strong influence on phase, amplitude, and spectral characteristics of the transmitted signal together with other perturbations introduced by the mobile cellular network (channel errors, background noise) (Chow et al., 1991). Moreover, the speech channel of the GSM is primarily intended for transmission of speech and DTMF signals only and not for transmission of digitally modulated arbitrary data. However, as the demand for different mobile communications services is continuously increasing, it is expected that an increasing number of mobile transactions will take place through the mobile cellular network. There exist three different GSM speech coders, which are referred to as the FR (full rate), HR (half rate) and EFR (enhanced full rate). Their corresponding European telecommunications standards are the GSM 06.10, GSM 06.20 and GSM 06.60, respectively (GSM 06.10 Full Rate (FR) Vocoder, 1996; GSM 06.20 Half Rate (HR) Vocoder, 1998; GSM 06.60 Enhanced Full Rate (EFR) Vocoder, 1997). These coders work on a 13 bit uniform PCM speech input signal, sampled at $8 \mathrm{kHz}$. The input is processed on a frame-by-frame basis, with a frame size of $20 \mathrm{~ms}$ (160 samples). A brief description of these coders follows.

\subsection{Full Rate Speech Coder}

The FR coder was standardized in 1987 (GSM 06.10 Full Rate (FR) Vocoder, 1996). This coder belongs to the class of Regular Pulse Excitation - Long Term Prediction linear predictive (RPE-LTP) coders. In the encoder part, a frame of 160 speech samples is encoded as a block of 260 bits, leading to a bit rate of $13 \mathrm{kbps}$. The decoder maps the encoded blocks of 260 bits to output blocks of 160 reconstructed speech samples. The GSM FR channel supports $22.8 \mathrm{kbps}$. Thus, the remaining $9.8 \mathrm{kbps}$ are used for error protection. The FR coder is described in GSM 06.10 down to the bit level and a set of digital test sequence for verification is also given (GSM 06.10 Full Rate (FR) Vocoder, 1996).

\subsection{Half Rate Speech Coder}

The HR coder standard was established to cope with the increasing number of subscribers. This coder is a $5.6 \mathrm{kbps}$ VSELP (Vector Sum Excited Linear Prediction) coder from Motorola (GSM 06.20 Half Rate (HR) Vocoder, 1998). In order to double the capacity of the GSM cellular system, the HR channel supports $11.4 \mathrm{kbps}$. Therefore, $5.8 \mathrm{kbps}$ are used for error protection. The measured output speech quality for the HR coder is slightly degraded to the quality of the FR coder. The normative GSM 06.06 gives the bit-exact ANSI-C code for this algorithm, while GSM 06.07 gives a set of digital test sequences for compliance verification (GSM 06.20 Half Rate (HR) Vocoder, 1998). 


\subsection{Enhanced Full Rate Speech Coder}

The EFR coder was the latest on field implemented. This coder is intended for utilization in the EFR channel, and it provides a substantial improvement in quality compared to the FR or HR coder. The EFR coder uses $12.2 \mathrm{kbps}$ for speech coding and $10.6 \mathrm{kbps}$ for error protection (GSM 06.60 Enhanced Full Rate (EFR) Vocoder, 1997). The speech coding scheme is based on Algebraic Code Excited Linear Prediction (ACELP). The bit exact ANSI-C code for the EFR coder is given in GSM 06.53 and the verification test sequences are given in GSM 06.54 (GSM 06.60 Enhanced Full Rate (EFR) Vocoder, 1997).

\subsection{Effects of the GSM Speech Coding on Transmission of Arbitrary Modulation Signals}

As mentioned before, the GSM system is primarily optimized to transmit the speech signal from one point to the other. If an arbitrary modulated signal is to be transmitted over the GSM, it must be in principal similar to the tempo-spectral characteristics of the speech signal (Bingham, 2000; Lipeika and Lipeikiene, 2008). Moreover, due to the characteristics of the RPE-LTP, VSELP and ACELP, the modulation signal should mimic the properties of the sustained voiced speech (pitch frequency and higher harmonics, formants, etc.) for best transmission properties (Bingham, 2000; Hanzo et al., 1999; Lehtonen, 2004). Due to the limited order of the autoregressive speech production modelling, the number of harmonics (i.e., carriers of the modulation signal) must not exceed a certain value (Lipeika and Lipeikiene, 2008). It is important also to mention the GSM speech coding effect on the theoretically maximal bit rate of the digitally modulated data. Namely, due to Shannon information theorem, the maximal bit rate of the digitally modulated data is limited with the available transmission bandwidth (Hanzo et al., 1999). In the case of GSM speech coders, this bandwidth directly corresponds to the bit rates of the FR, HR, and EFR speech coders (see Subsections 2.1, 2.2, and 2.3). Spectrum efficiency of the GSM transmission system is additionally increased through the use of Discontinuous Transmission (DTX), switching the transmitter on only during speech activity periods. The use of DTX is associated with potential degradation of the arbitrary modulated signal quality due to signal clipping (modulation signal detected as noise) and noise contrast effects (Scourias, 1995). It is thus expected that the use of DTX has a negative impact on the performance of the transmission of arbitrary modulated data through the GSM system. VAD (Voice Activity Detection) is used to decide upon presence of active speech (ETSI EN 300730 v7.0.1, 2000). To reduce the annoying modulation of the background noise at the receiver (noise contrast effects), CNG (Comfort Noise Generation) is used, inserting a coarse reconstruction of the background noise at the receiver. Additionally, GSM incorporates also nonstandard Echo Cancellation algorithm. The echo canceller's basic function is to eliminate all the signals, which are not similar to speech, and the sub signals, which appear along the speech transmission (Eneroth, 2001). All three GSM coders described include the functions of DTX, VAD, CNG and Echo Cancellation. For the mobile transaction system the robustness and reliability 
of the digital data transmission over the GSM speech channel would have a very strong priority over the transmission speed. Namely, the transmission bit rate of $250 \mathrm{bps}$ would be more than enough if the superior transmission robustness can be achieved at such data bit rate.

\section{Digital Modulation Techniques}

There are many different digital modulation techniques that can be used to transmit the digital data over various media such as radio waves, acoustic waves (in the air, or water), telephone cable pairs, mains power supply installations, etc. However, not all digital modulations are appropriate to transmit digital data over a very specific media such as speech channel of GSM or any other mobile communication system. In this section we will review typical modulation techniques and discuss their appropriateness for the mentioned task. There are three basic digital modulation techniques: ASK (Amplitude Shift Keying), FSK (Frequency Shift Keying), and PSK (Phase Shift Keying) (Xiong, 2006). Along with these basic modulations, there is also a wide range of coherent and noncoherent, singlecarrier and multi-carrier modulation techniques which are basically combinations (so called hybrids), or elaborates of the above mentioned basic modulations. Some of these are QAM (Quadrature Amplitude Modulation), DPSK/BPSK (Differential/Binary Phase Shift Keying), QPSK (Quadrature Phase Shift Keying), GMSK (Gaussian-filtered Minimum Shift Keying), OFDM (Orthogonal Frequency Division Multiplexing) and CDMA (Code Division Multiple Access) (Batra et al., 2004; Schmidl et al., 1997; Schmidt and Jondral, 2003; Wiegandt et al., 2003; Xiong, 2006; Zhengdao and Giannakis, 2000). Due to the specific property of the frame-based speech coding system, which does not assure arbitrary phase-shifting preservation, the coherent, as well as phase-based modulation techniques can not be utilized to transmit modulated data over the speech channel of the GSM system. The main reason why GSM speech coders do not support arbitrary phase shifting lies in asynchronous windowing of the input speech or modulation signal inside the RPE-LPT (Hanzo et al., 1999). The remaining options are ASK, FSK, and OFDMbased modulations. In the next sections a detailed description of a novel PCCD-OFDMASK (multi-carrier phase-continuous context-dependent orthogonal frequency division multiplexing amplitude shift keying) will be provided along with the performance comparison to the single-carrier ASK and FSK.

\section{Description of Proposed Digital Modulation Technique PCCD-OFDM-ASK}

This section describes details about the proposed multicarrier modulation technique PCCD-OFDM-ASK. First, a short overview of the basic OFDM system will be provided.

\subsection{Basic OFDM Principles}

OFDM (Orthogonal Frequency Division Multiplexing) is very similar to the well-known technique of FDM (Frequency Division Multiplexing). OFDM uses the principles of 
FDM to allow multiple messages to be sent over a single transmission channel (Gurprakash and Arokiaswami, 2003; Huang et al., 2003; Lawrey, 2001; Schmidl et al., 1997). OFDM can be simply defined as a form of multicarrier modulation where the carrier spacing is carefully selected so that each subcarrier is orthogonal to the other subcarriers. As is well known, orthogonal signals can be separated at the receiver by correlation techniques; hence theoretically, intersymbol interference among channels can be eliminated (Lawrey, 2001). Orthogonality can be achieved by carefully selecting carrier spacing, such as letting the carrier spacing be equal to the reciprocal of the useful symbol period (Schmidl et al., 1997; Wiegandt et al., 2003; Zhengdao and Giannakis, 2000). Eq. (1) presents a continuous-time set of orthogonal sinusoids, which represent the subcarriers of an unmodulated real OFDM signal.

$$
s_{k}(t)= \begin{cases}\sin \left(2 \pi k f_{0} t\right), & 0<t<T, k=1,2, \ldots, M, \\ 0, & \text { otherwise }\end{cases}
$$

where $f_{0}$ is the carrier spacing, $M$ is the number of carriers and $T$ is the symbol period. Since the highest frequency component is $M f_{0}$, the transmission bandwidth is also $M f_{0}$. These subcarriers are orthogonal to each other, because multiplying the waveforms of any two subcarriers and integrate over the symbol period gives the result zero. Multiplying the two sine waves together is the same as mixing these sub carriers (Huang et al., 2003; Schmidl et al., 1997; Zhengdao and Giannakis, 2000). The orthogonality property of OFDM signals is reflecting also to its spectrum. In the frequency domain each OFDM subcarrier has a sinc $(\sin (x) / x)$ frequency response, as shown in Fig. 3. This is a result of the symbol time corresponding to the inverse of the carrier spacing (Wiegandt et al., 2003).

As far as the receiver is concerned, each OFDM symbol is transmitted for a fixed time ( $\left.T_{F F T}\right)$ with no tapering at the ends of the symbol. This symbol time corresponds to the inverse of the subcarrier spacing of $1 / T_{F F T} \mathrm{~Hz}$ (Ibars and Bar-Ness, 2001). The rectangular waveform in the time domain results in a sinc frequency response in the frequency domain. The sinc shape has a narrow main lobe, with many side-lobes that decay slowly with the magnitude of the frequency difference away from the centre. Each carrier has a peak at the centre frequency and nulls evenly spaced with a frequency gap equal to the carrier spacing (Bingham, 2000; Lehtonen, 2004). When this signal is detected (demodulated) using a FFT (Fast Fourier Transform) the spectrum is not continuous as shown in in Fig. 3, but has discrete samples. If the FFT is time synchronized, the frequency samples of the FFT correspond to just the peaks of the subcarriers, thus the overlapping frequency region between subcarriers does not affect the receiver. The measured peaks correspond to the nulls for all other subcarriers, resulting in orthogonality between the subcarriers. The real advantage of the OFDM lies in its simplicity and computational efficiency. Namely, the set of orthogonal carriers defined with Eq. (1) can be constructed using IFFT (Inverse FFT) (Wiegandt et al., 2003). The modulation procedure is as follows: each of the data subcarriers is set to a magnitude and phase based on the digital data being sent and the modulation scheme; all unused subcarriers are set to zero. This sets up the OFDM signal in the frequency domain. An IFFT is then used to convert this 

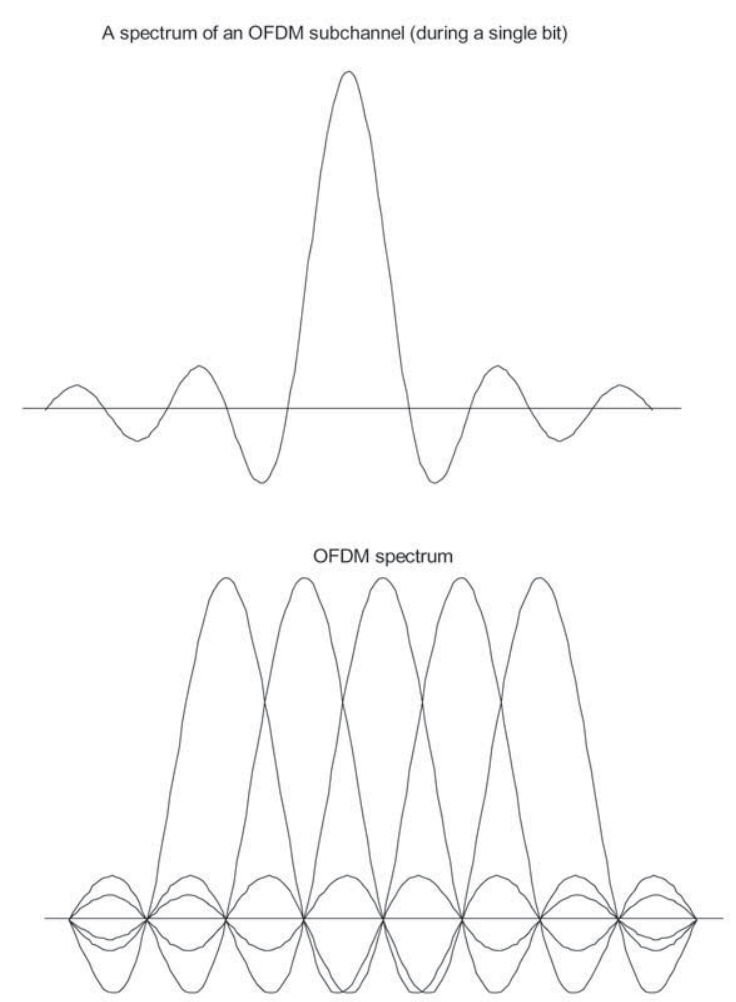

Fig. 3. Frequency response of single OFDM carrier and five mixed OFDM carriers.

signal to the time domain, allowing it to be transmitted. In the frequency domain, before applying the IFFT, each of the discrete samples of the IFFT corresponds to an individual subcarrier. Most of the subcarriers are modulated with data. The outer subcarriers are unmodulated and set to zero amplitude. These zero subcarriers provide a frequency guard band before the Nyquist frequency. The OFDM demodulation procedure uses actually an inverse scheme: the FFT is used to detect the magnitude and phase of each subcarrier. The actual digital data demodulation depends on the modulation scheme applied at the modulator (Wiegandt et al., 2003).

\subsection{Data Transmission Parameters of Proposed PCCD-OFDM_ASK Modulation Scheme}

Most important two parameters of each digital data transmission system are the bit rate $r$ and required frequency bandwidth $f_{B}$. Since the OFDM is a multicarrier modulation scheme, a symbol rate $m$ could also be defined (Sun, 2001). The input digital data which have to be modulated will be grouped into octets $D_{T}[m]$ of 8 bits (1 octet $D$ represents 1 Byte of data) (Fig. 4). Each symbol will therefore consist of $M=8$ carriers. Each carrier will comprehend information about the value of one bit from the octet. In proposed 


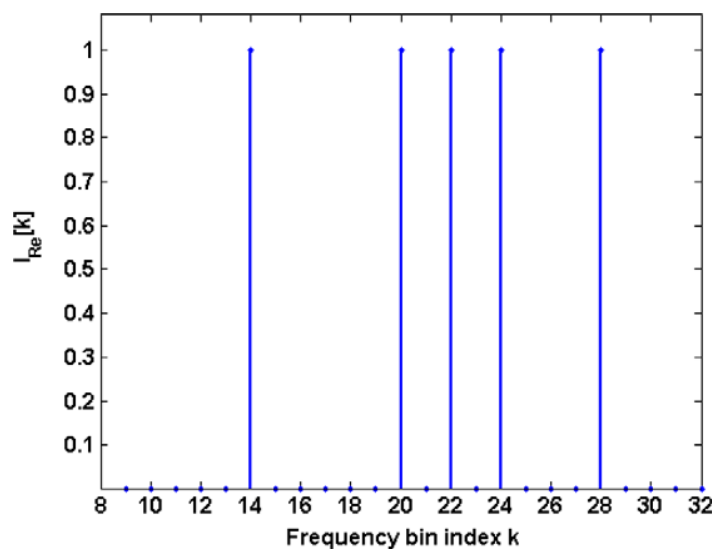

Fig. 4. Carrier modulation principle for the octet $D_{T}[m]=10011101$.

modulation scheme the grouping of 16 bits into one symbol (utilization of 16 sinusoidal carriers) is also possible.

The target bit rate of proposed basic PCCD-OFDM-ASK modulation scheme will be $r=250 \mathrm{bps}$. The symbol rate $p$ is therefore defined as

$$
p=\frac{r}{M}=\frac{250 \mathrm{bit} / \mathrm{s}}{8 \mathrm{bit} / \mathrm{symb}}=31.25 \mathrm{symb} / \mathrm{s}
$$

As mentioned in Section 2, The sampling rate $f_{S}$ of $8 \mathrm{kHz}$ is applied in the GSM coders. The length (in samples) of each symbol period $T_{M}$ is therefore defined as follows:

$$
T_{M}=\frac{f_{S}}{p}=\frac{8000 \mathrm{samples} / \mathrm{s}}{31.25 \mathrm{symb} / \mathrm{s}}=256 \mathrm{samples} / \mathrm{symb} .
$$

Since the OFDM uses IFFT/FFT operations for modulation/demodulation purposes (Ibars and Bar-Ness, 2001), a FFT order of FFT_Ord = 256 will be applied as deduced from Eq. (3). The minimal carrier spacing $f_{0 M I N}$ is then defined as

$$
f_{0 M I N}=\frac{f_{S}}{F F T \_O r d}=\frac{8000 \mathrm{~Hz}}{256}=31.25 \mathrm{~Hz} .
$$

The minimal required bandwidth $f_{B M I N}$ can be computed using minimum carrier spacing $f_{0 M I N}$ and number of carriers $M$ as

$$
f_{B M I N}=M f_{0 M I N}=8 \cdot 31.25 \mathrm{~Hz}=250 \mathrm{~Hz}
$$

The allowed frequency bandwidth in presented mobile payment system based on data transmission over the GSM speech channel is $f_{B}=500 \mathrm{~Hz}$ (data transmission from the terminal to the center). The signal transmission over the GSM channel is a subject to various linear and non-linear distortions (see Subsection 2.4). In order to get the modulation signal with similar time-spectral characteristics as sustained voiced speech, and 
Table 1

Carrier frequency definitions of proposed PCCD-OFDM-ASK modulation scheme

\begin{tabular}{ccc}
\hline $\begin{array}{c}\text { Carrier } \\
\text { number } m\end{array}$ & $\begin{array}{c}\text { Frequency bin index } k \\
\text { (FFT order }=256)\end{array}$ & $\begin{array}{c}\text { Carrier } \\
\text { frequency } f_{k}[\mathrm{~Hz}]\end{array}$ \\
\hline 1 & 14 & 437.5 \\
2 & 16 & 500.0 \\
3 & 18 & 562.5 \\
4 & 20 & 625.0 \\
5 & 22 & 687.5 \\
6 & 24 & 750.0 \\
7 & 26 & 812.5 \\
8 & 28 & 875.0 \\
\hline
\end{tabular}

to simultaneously decrease the inter-carrier interferences while maintaining the total allowed bandwidth of $f_{B}=500 \mathrm{~Hz}$, a two-times broader carrier spacing of $f_{0}=62.5 \mathrm{~Hz}$ has been selected, just occupying every second frequency bin in IFFT/FFT. Since the lowest standardized cut-off frequency of the voice transmission over GSM voice channel is defined around $300 \mathrm{~Hz}$, the starting frequency for proposed modulation scheme will be slightly higher: at $f_{1}=437.5 \mathrm{~Hz}$. The actual carrier frequencies $f_{k}$ in $\mathrm{Hz}$ are computed as follows:

$$
f_{k}=k \cdot \frac{f_{S}}{F F T_{-} \text {Ord }}, \quad \text { where } k=14,16, \ldots, 28 .
$$

Table 1 presents the carrier frequency definitions and FFT frequency bin indexes applied in the proposed modulation scheme using 8 data carriers. It should be noted, that the number of used carriers can be arbitrary. For example, if bit rates up to 500 bps are needed, there are 16 carriers applied in the proposed modulation scheme.

\subsection{PCCD-OFDM-ASK Carrier Modulation Procedure}

The time-domain real modulation signal $s_{T}[n]$ is computed separately for every data symbol $D_{T}[m]$, carrying one byte of digital data, using the IFFT:

$$
s_{T}[n]=\underset{F F T_{-} O r d}{I F F T}\left\{I_{\operatorname{Re}}[k], Q_{\operatorname{Im}}[k]\right\}, \quad \text { where } k=1, \ldots, F F T_{-} O r d .
$$

The length of the modulation signal $s_{T}[n]$ is $T_{M}$ samples. The imaginary $Q_{I m}[k]$ and real $I_{R e}[k]$ components of the IFFT are defined on the basis of $D_{T}[m]$ as follows. First, the imaginary component are set to zero:

$$
Q_{\operatorname{Im}}[k]=0, \quad \text { where } k=0, \ldots, F F T \_O r d .
$$

Usually, some bandwidth is lost by setting the imaginary component to zero, but because of unique transmission influences that are present, when sending data over GSM 
voice channel, this is unfortunately necessary. The actual digital input data $D_{T}[m]$ is modulated onto the real component $I_{R e}[k]$.

$$
I_{\mathrm{Re}}[k]= \begin{cases}1, & \text { if } D[m]=1, \\ 0, & \text { if } D[m]=0,\end{cases}
$$

where $k=2 m+12$, and $m=1,2, \ldots, 8$.

For all other values of $k=1, \ldots, F F T \_O r d$, not defined in Eq. (9), the $I_{R e}[k]$ is set to zero. Note that the conjugate-symmetry of vector-pair $I_{R e}[k], Q_{I m}[k]$ must be assured prior to IFFT operation in order to get the real output sequence $s_{T}[n]$. Fig. 4 presents an example of data modulation principle. The resulting time-domain modulation signal $s_{T}[n]$ after IFFT is presented in Fig. 5 . The modulation signal $s_{T}[n]$, corresponding to one data symbol, can have abrupt changes in sample values at its beginning or ending. In order to prevent unwanted spectral-spreading (due to Dirac-like pulses in the time-waveform of $s_{T}[n]$ ), a special signal shaping must be applied at the beginning and ending of each symbol $s_{T}[n]$ (Gurprakash and Arokiaswami, 2003). This signal shaping is performed in classical OFDM in the manner of so called guard periods and cyclic prefix (Lawrey, 2001), but these techniques are not adequate for this kind of transmissions. In proposed modulation scheme a root-raised cosine windowing is performed. The root-raised cosine window $w_{R R C}[n]$ is defined as

$$
w_{R R C}[n]=\left(\cos \left(2 \pi N \frac{N+n-1}{2 N-1}\right)\right)^{2}, \quad n=1,2, \ldots, N,
$$

where $N$ defines the length of the $w_{R R C}[n]$ in samples, typically $N=20$. First and the last $N$ samples of the $s_{T}[n]$ are multiplied with $w_{R R C}[n]$ or its time-reversed values respectively, while the most of the main part remains as it is. The modulation signal $s_{T}[n]$ multiplied with proposed shaping window is depicted in Fig. 6.

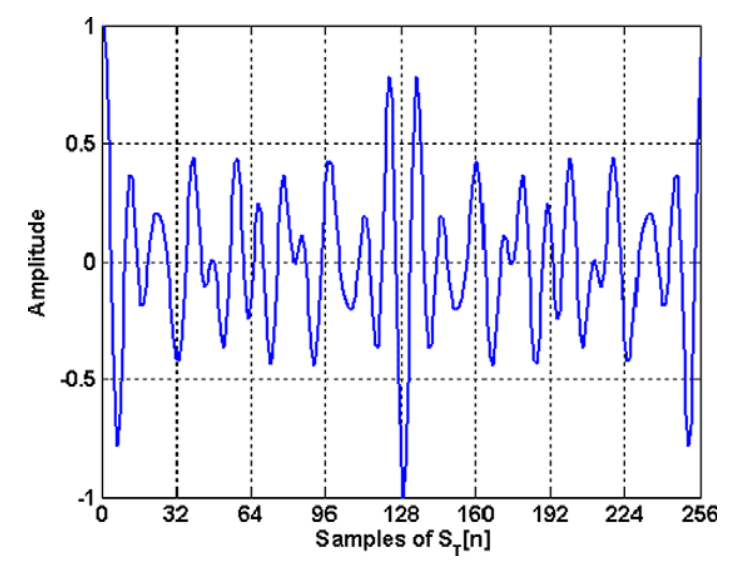

Fig. 5. Corresponding time-domain modulation signal $s_{T}[n]$ as a result of IFFT. 


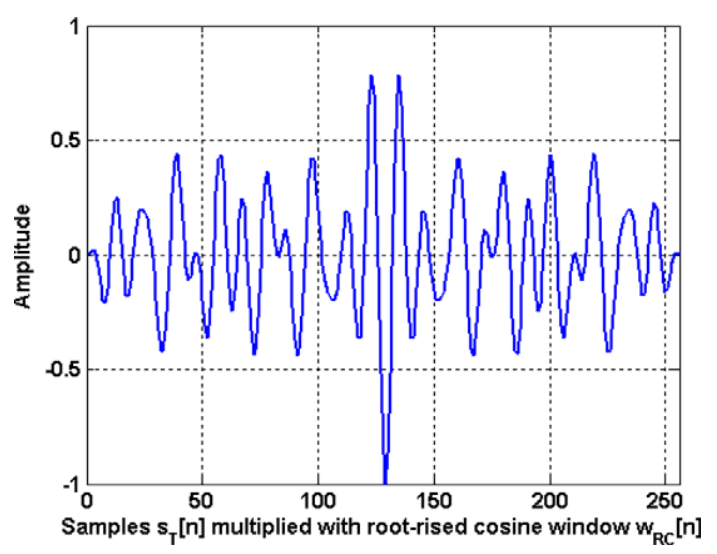

Fig. 6. Modulation signal $s_{T}[n]$ multiplied with the proposed root-rised cosine window $w_{R R C}[n]$.

\subsection{Phase-Continuity and Context Dependency Principles (PCCD)}

One of the most important improvements of proposed PCCD-OFDM modulation scheme with respect to classical OFDM is the utilization of the phase-continuity and context dependency principle. The PCCD principle refers to the carrier modulation of each data symbol. Namely, the phase of the modulated carrier $m$ in the frame $t$ depends on the modulated carrier $m$ in the previous frame $t-1$ as well as on the modulated carrier $m$ in the following frame $t+1$, for each carrier $m=1,2, \ldots, M$. So in case that, the bit $D[m]$ is not changed from the data symbol in the time instant $t-1$ to $t$, then the phase of the corresponding modulated carrier $m$ is preserved from symbol $t-1$ to $t$. In this case there is no need to apply root-raised windowing for all modulation carriers. Phase-continuity between data symbols is essential for achieving smaller influences by codec and consequently better data transmissions. IFFT doesn't care about phase continuity between separate symbols and because of that a look-up table with sample data of

Table 2

PCCD modulation principle

\begin{tabular}{cc}
\hline $\begin{array}{c}\text { Bit } D[m] \text { transition in symbols: } \\
\text { previous }(t-1)-\text { current }(t)-\text { next }(t)\end{array}$ & $\begin{array}{c}\text { Root-rised window usage on carrier } \\
m \text { in the current symbol } t:\end{array}$ \\
\hline $0-\mathbf{0}-0$ & none \\
$0-\mathbf{0}-1$ & none \\
$0-\mathbf{1}-0$ & Start $\left(w_{R R C}[n]\right)+$ End (time reversed $\left.w_{R R C}[n]\right)$ \\
$0-\mathbf{1}-1$ & Start $\left(w_{R R C}[n]\right)$ \\
$1-\mathbf{0}-0$ & none \\
$1-\mathbf{0}-1$ & none \\
$1-\mathbf{1}-0$ & End (time reversed $w_{R R C}[n]$ \\
$1-\mathbf{1}-1$ & none \\
\hline
\end{tabular}


one period sinus waveform is applied to generate orthogonal carrier signals and preserve phase through symbols. However, if a particular bit is changed from the previous symbol to the current one, then a root-raised windowing of the corresponding carrier must be performed. Table 2 explains the PCCD modulation principle. Figs. 7 and 8 present a spectrogram comparison of modulation signal $s_{T}[n]$ without and with proposed rootraised cosine windowing $w_{R R C}[n]$.

It is evident that the proposed symbol shaping substantially reduces unwanted spectral spreading at symbol borders. The application of root-raised cosine window on modulated carrier at bi transitions "0-to-1":

$$
s_{T}[n]=\prod_{n=1}^{20} w_{R R C}[n] \cdot s_{T}[n] .
$$

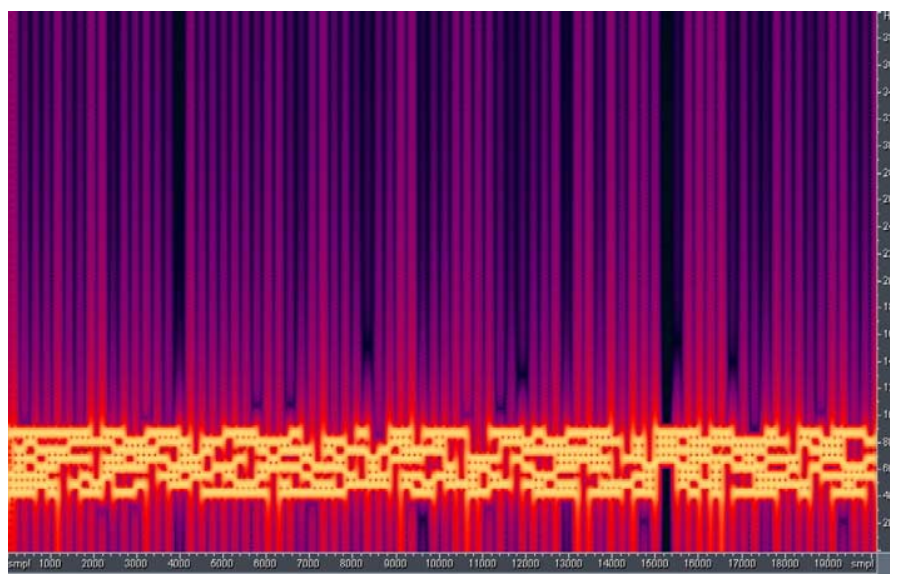

Fig. 7. Spectrogram of modulation signal $s_{T}[n]$ without root-rised weighting.

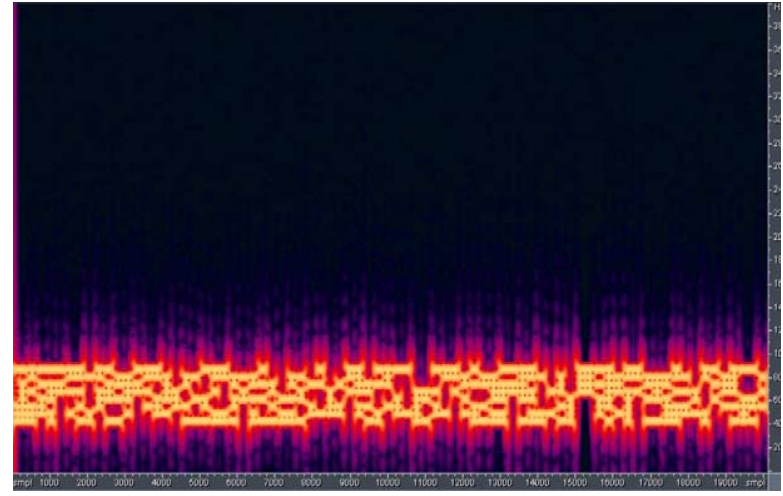

Fig. 8. Spectrogram of modulation signal $s_{T}[n]$ with proposed root-rised weighting $w_{R R C}[n]$. 
The application of $w_{R R C}[n]$ at bit transition "1-to-0" (time reversal) is as follows:

$$
s_{T}[n]=\prod_{n=F F T_{-} O r d-19}^{F F T \_ \text {Ord }} w_{R R C}\left[F F T \_O r d+1-n\right] \cdot s_{T}[n] .
$$

\subsection{Signal Synchronization}

The quality and the performance of the PCCD-OFDM-ASK demodulation strongly depend on the signal synchronization.. The demodulation is performed using the FFT which requires a perfectly time-aligned frame of the modulation signal at the input (Schmidl et al., 1997; Zhengdao and Giannakis, 2000). The frame of the signal should correspond to one symbol $s_{T}[n]$. If the symbol alignment at the demodulator side is lost, the demodulation will produce wrong results. Therefore the modulation signal itself should contain such synchronization sequences that very accurate automatic detection of symbols is possible. OFDM usually uses cyclic prefix for synchronization, while other more simple techniques are also used such as combined simple sinusoids at predetermined frequencies that can be detected with a set of band pass filters. Such techniques can't be used in data transmission over GSM voice channel. In order to achieve precise signal synchronization, a special chirp synchronization signal $g[n]$ is constructed. The proposed chirp signal $g[n]$ consists of two sinusoidal waveforms whose frequency is linearly increasing in time:

$$
g[n]=0.5 \sin \left(2 \pi\left(f_{1}+\frac{\lambda n}{f_{S}}\right) \frac{n}{f_{S}}\right)+0.5 \sin \left(2 \pi\left(f_{2}+\frac{\lambda n}{f_{S}}\right) \frac{n}{f_{S}}\right),
$$

where $f_{S}$ is a sampling frequency $(8 \mathrm{kHz}), f_{1}=400 \mathrm{~Hz}$ and $f_{2}=650 \mathrm{~Hz}$ are the starting frequencies of the two sinusoidal signals, and $\lambda=2000$ is a rate of frequency increase (chirp rate). The length of the chirp signal $g[n]$ is 640 samples $(n=1,2, \ldots, 640)$. The start and the tail of the chirp signal is multiplied with the above defined root-raised cosine window $w_{R R C}[n]$ in order to prevent unwanted spectral spreading (similar to symbol shaping). The spectrogram of the proposed synchronization signal is presented in Fig. 9.

The used property of the chirp signal $g[n]$ is correlation. The signal $g[n]$ is well correlated only with itself. Moreover, the autocorrelation produces high values only at full alignment of the two chirp signals. The cross correlation of $g[n]$ with any other arbitrary signal produces very low values. This property is shown in Fig. 10.

At the modulator site, the synchronization sequences $g[n]$ are inserted at prescribed positions (i.e., after every 32nd symbol). At the demodulator, the correlation of $g[n]$ and $s_{T}[n]$ is computed. High value of $<g[n], s_{T}[n]>$ indicates the exact position of the synchronization sequence (see Fig. 10). The proposed demodulation scheme comprehends also the adaptation procedure to set the decision threshold for particular carrier magnitudes. Therefore, special measuring sequences (described in the next Subsection 4.6) are inserted in the modulation signal at the modulator. The measuring sequences are marked by the time reversed chirp (see Fig. 11). 


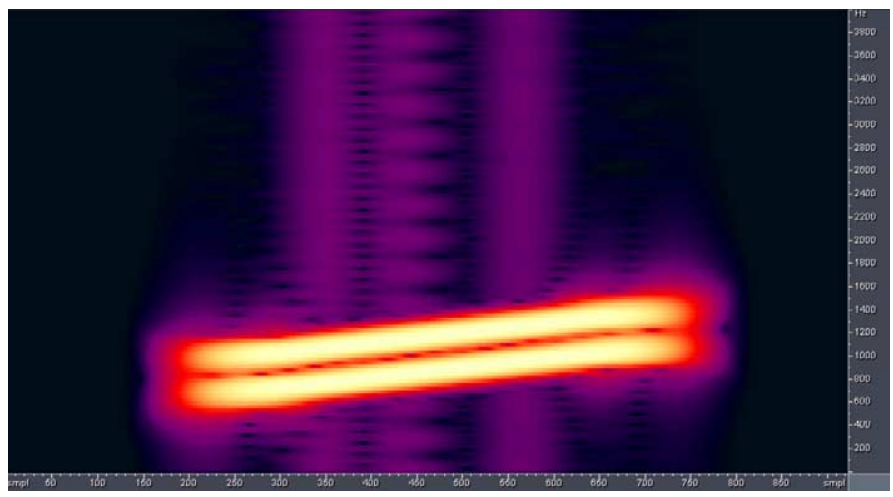

Fig. 9. Spectrogram of the proposed synchronization chirp signal $g[n]$.

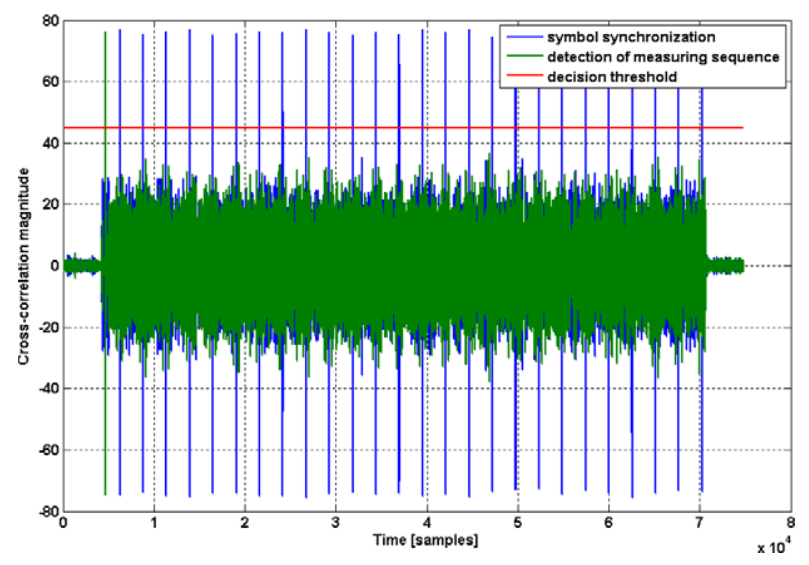

Fig. 10. Symbol synchronization principle using the chirp signal.

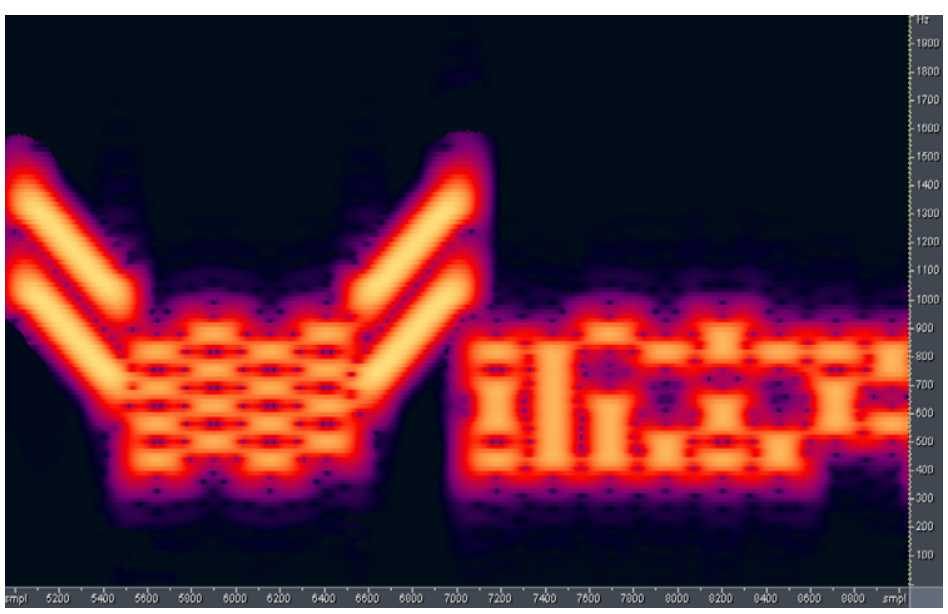

Fig. 11. Alternating measuring bit sequence for modulation scheme with 8 carriers. 
The synchronization procedure is performed at the demodulator as follows; First the cross-correlation $x_{1}[n]$ between the prototype chirp signal $g[n]$ and the input modulation signal $s[n]$ is performed:

$$
x_{1}[n]=\sum_{i=1}^{640} s[n+i] \cdot g[i]
$$

Next, the cross-correlation $x_{2}[n]$ between the time reversed chirp and the input modulation signal is computed:

$$
x_{2}[n]=\sum_{i=1}^{640} s[n+i] \cdot g[641-i] .
$$

In order to produce the energy-normalized cross-correlation estimates, the energies of the input modulation signal ES and the chirp signal EC are computed as follows:

$$
E_{S}=\frac{1}{640} \sqrt{\sum_{i=1}^{640}(s[n+i])^{2}}, \quad E_{C}=\frac{1}{640} \sqrt{\sum_{i=1}^{640}(g[i])^{2}} .
$$

Next, the energy-normalized cross correlation estimates $X_{1}[n]$ and $X_{2}[n]$ are determined:

$$
X_{1}[n]=\frac{x_{1}[n] \cdot E_{C}}{1+E_{S}}, \quad X_{2}[n]=\frac{x_{2}[n] \cdot E_{C}}{1+E_{S}} .
$$

In the last step, the candidates of the positions of the synchronization sequences are searched:

$$
\begin{aligned}
& \text { AllPeaks }[n]= \begin{cases}1, & \text { if } X_{1}[n] \geqslant G, \\
0, & \text { if } X_{1}[n]<G ;\end{cases} \\
& \text { AllPeaksMeasure }[n]= \begin{cases}1, & \text { if } X_{2}[n] \geqslant G, \\
0, & \text { if } X_{2}[n]<G,\end{cases}
\end{aligned}
$$

where $G$ represents the a priori determined decision threshold. Unfortunately, this threshold has to be determined empirically. The good choice of $G$ is: $40<G<80$. Finally, the most probable synchronization peak is searched using simple local-maximum peak search procedure.

\subsection{PCCD-OFDM-ASK Adaptive Magnitudes}

In PCCD-OFDM ASK each bit in symbol is presented with presence or absence of individual orthogonal carrier (Larsson et al., 2001). Decision for each bit on demodulator side is based on size of calculated magnitude for individual carrier. These magnitudes are strongly dependent on signal interferences that are occurring during transmission 
(Nikolic and Peric, 2008). Consequence is that in some conditions it might occur that calculated magnitude for one carrier can mean bit value ' 0 ', but for other carrier it could mean bit value ' 1 '. For solving this problem, modulator needs to send additional measuring sequence at start and between the transmissions so that demodulator adapts new magnitudes for each orthogonal carrier. PCCD-OFDM-ASK is using special chirp signals to detect start and end of data frames, as already described.

Demodulator must know the difference between data frames and additional measuring sequences. Each measuring sequence starts with reversed chirp signal followed by 4 symbols with alternating bit sequence (Table 3 and Fig. 11).

With predefined bit values in measuring sequence, demodulator can adapt measured magnitudes and use them as a reference for further decisions. However, because of possible interferences in measuring sequence, predefined magnitude references are also used for proper decisions.

Table 3

Alternating measuring bit sequence for modulation scheme with 8 carriers

\begin{tabular}{ccccc}
\hline Carrier number $m$ & Symbol 1 & Symbol 2 & Symbol 3 & Symbol 4 \\
\hline 1 & 1 & 0 & 1 & 0 \\
2 & 0 & 1 & 0 & 1 \\
3 & 1 & 0 & 1 & 0 \\
4 & 0 & 1 & 0 & 1 \\
5 & 1 & 0 & 1 & 0 \\
6 & 0 & 1 & 0 & 1 \\
7 & 1 & 0 & 1 & 0 \\
8 & 0 & 1 & 0 & 1 \\
\hline
\end{tabular}

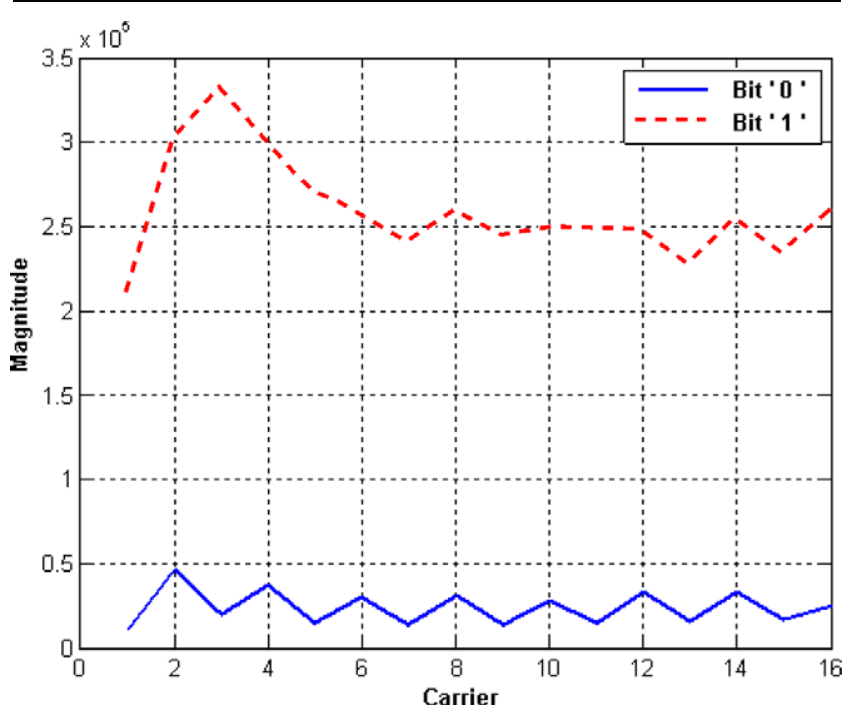

Fig. 12. Alternating measuring bit sequence for modulation scheme with 8 carriers. 
Demodulator gives two parallel results; one is based on predefined magnitudes and other on adaptive magnitudes. Predefined magnitudes were calculated on mass demodulation results with different mobile phones in variety of environments and all three GSM coders.

Fig. 12 presents these predefined magnitudes for just one of the PCCD OFDM ASK modulation schemes with 16 carriers per symbol, starting frequency of first carrier at $437.5 \mathrm{~Hz}, 256$ samples per symbol and GSM-EFR coder.

\subsection{PCCD-OFDM ASK Normalization}

Strength of the received modulation signal can vary a lot in a GSM transmission system. Therefore, the demodulator must adapt to these amplitude variations in order to successfully demodulate the data from the carrier magnitudes. One of the most frequent causes for these signal-amplitude variations is the changing distance between the terminal speaker and the microphone of the mobile phone. Second cause is AGC (Automatic Gain Control) that is located at most mobile phones. Purpose of AGC is to increase or decrease

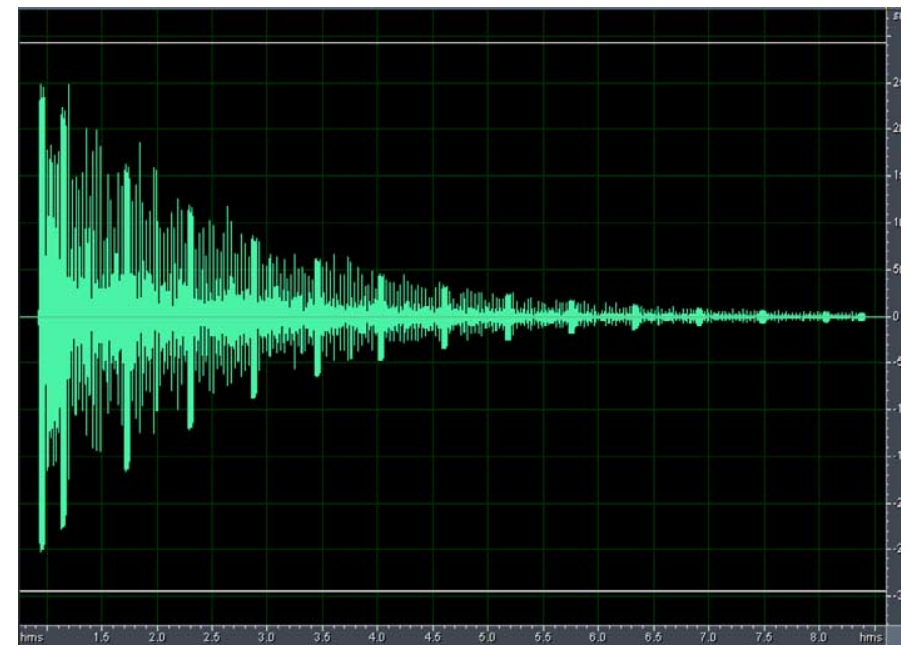

Fig. 13. Example of signal with controlled amplitude variations for magnitude normalization test.

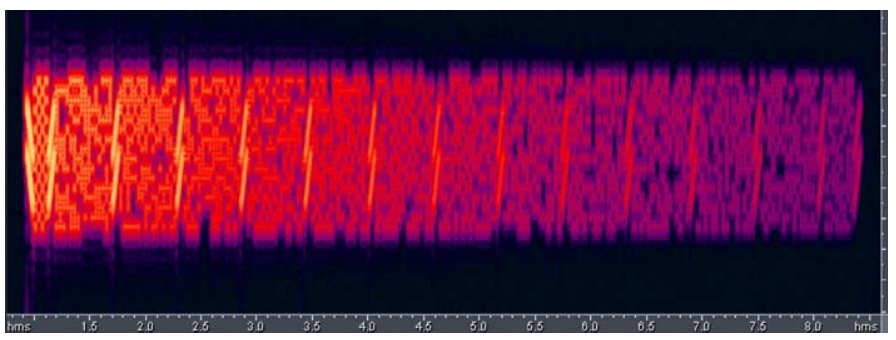

Fig. 14. Spectrogram of signal in Fig. 13. 
received signal to improve communication quality. Decision for each bit on demodulator side is based on the value of calculated magnitude for individual carrier. Therefore amplitude normalization of received signal must be made before final bit decisions. The solution lays in previously described chirp synchronization, and energy normalizations (Eqs. (16) and (17)). The proportion between the energies of the input modulation signal $E_{S}$ and the chirp signal $E_{C}$ is adaptively calculated and used for multiplying with each symbol that is located after used chirp signal, till next chirp signal, where new normalization is carried out.

Figs. 13 and 14 are presenting example of controlled amplitude variations where adaptive normalization is carried out and all bit decisions that are based on magnitude size, are calculated correctly. Fig. 13 represents the modulation signal in the time domain whereas Fig. 14 represents the spectrogram of the modulation signal.

\subsection{PCCD-OFDM Carrier Detection and Digital Data Retrieval}

Signal filtering, chirp synchronization, adaptive magnitudes calculation and normalization are the first processing steps that are applied on the received signal. Next step of the demodulation procedure is separating signal into individual symbols using the synchronization data (see Subsection 4.3). The OFDM demodulation procedure uses FFT on these symbols for magnitude calculation of individual carriers that were already presented in Table 1. Usually, Hamming or Hann windowing, is applied on the time-domain signal before the FFT. The windowing is important because it reduces wraparound leakage. However, the properties of the transformed signal can vary much due to different windowing. Some windows are designed for general purpose FFT, while the others are strongly specialized. In controversy, the proposed PCCD-OFDM demodulator applies just a simple square (boxcar) windowing, which results in a spectrum with the narrowest main central lobe width and slightly higher magnitudes of the side lobes. This procedure provides the highest possible frequency resolution whereas the high magnitudes of the side frequency lobes do not affect the quality of the demodulation. A result magnitude of individual carrier does not contain discrete values. Fig. 15 presents example of demodulation principle on symbol with bit sequence "0101001000101000". Decision of demodulator for individual carrier bit value $B V$ is based on comparison between individual calculated magnitude $M$ and individual decision thresholds $D T$.

$$
B V[n]= \begin{cases}1, & \text { if } M[n] \geqslant D T[y], \\ 0, & \text { if } M[n]<D T[y] .\end{cases}
$$

$D T$ is a set of decisions thresholds that is calculated with zero magnitude thresholds $Z M$, one magnitude thresholds and decision values $D V$. Zero and one magnitudes are a result of predefined and adapted magnitudes, while decisions factors are constants.

$$
\begin{aligned}
& D T[y]=\frac{Z M[n][t]+O M[n][t]}{D V[x]}, \\
& n=1,2,3, \ldots, 16, \quad t= \begin{cases}1, & \text { predefined mag. } \\
2, & \text { adapted mag. }\end{cases}
\end{aligned}
$$




$$
x=1,2,3, \ldots, 8, \quad y=t n+x .
$$

Decision values are 8 independent values that are result of different offline testing where the main goal was to embrace different signal fluctuations (Table 4).

\subsection{PCCD-OFDM ASK System Overview}

The simplified block diagram of the proposed PCCD-OFDM-ASK modulation/demodulation system is presented in Fig. 16. Both platforms; terminal and center are processing not just modulation and demodulation, but also other advanced algorithms such as BEC (backward error correction), FEC (forward error correction) and ECC (elliptic curve cryptography) (Davis and Jedwab, 1997). All this techniques are running in both ways (full-duplex operation), simultaneously in real time. Besides these algorithms that are important for proper data transmission and security, other process are also carried out such as GUI support (graphical user interface) on terminal, center communication with

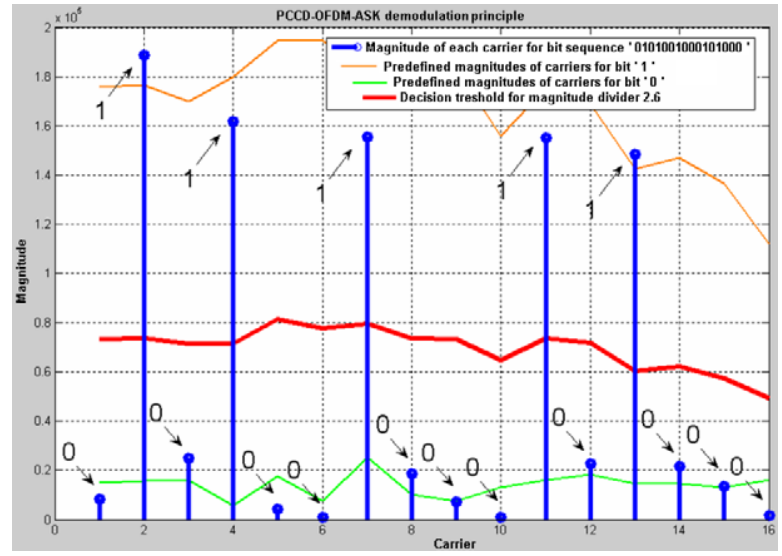

Fig. 15. Alternating measuring bit sequence for modulation scheme with 8 carriers.

Table 4

Decision factors

\begin{tabular}{cc}
\hline Number $x$ & Decision values \\
\hline 1 & 1.4 \\
2 & 1.7 \\
3 & 2.0 \\
4 & 2.3 \\
5 & 2.6 \\
6 & 2.9 \\
7 & 3.1 \\
8 & 3.4 \\
\hline
\end{tabular}




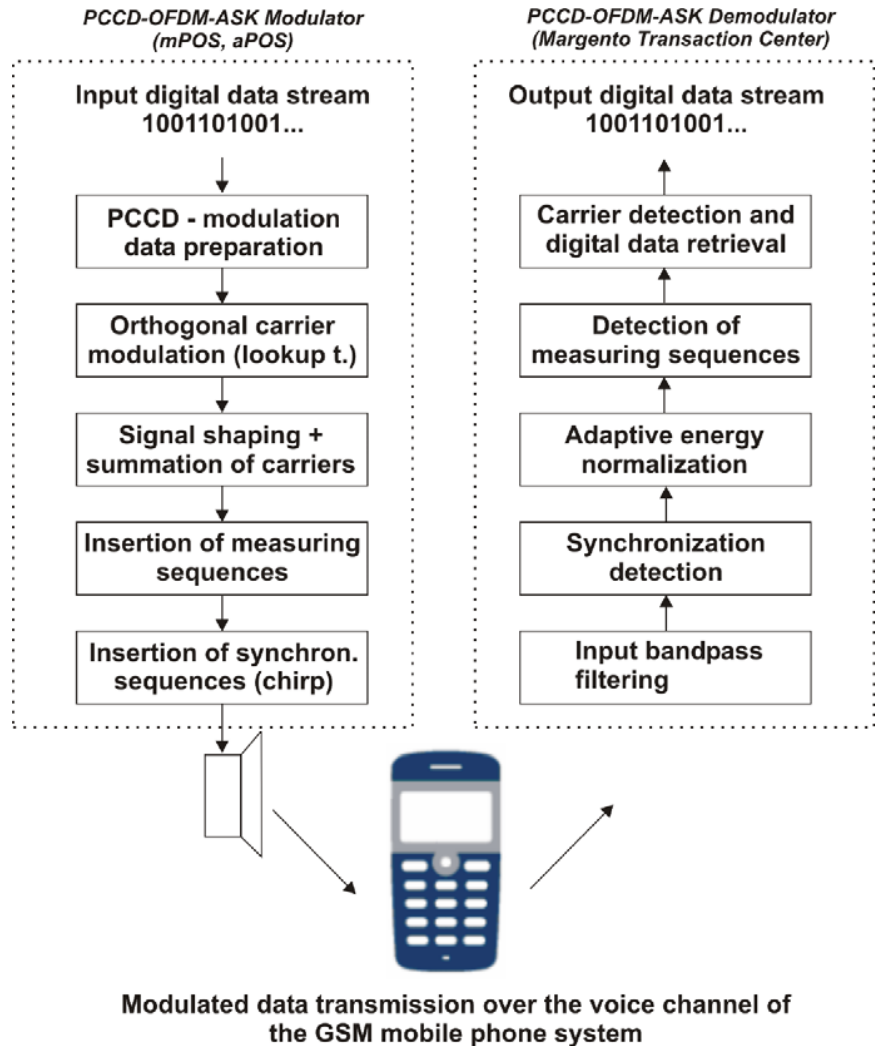

Fig. 16. Block diagram of proposed PCCD-OFDM-ASK modulation/demodulation scheme.

financial institutions, center communication with mobile operator switching center, etc. The main difference in processing, between center and terminal, is that center is communicating in parallel with many terminals and because of that more processing power is needed.

\section{Verification with Comparison}

Verification of PCCD OFDM ASK was made in four steps. Through these steps we have come to conclusions which parameters for PCCD OFDM ASK are most appropriate for transmitting signal over GSM voice channel and how did new modulation technique perform toward old ones (standard ASK and FSK) that were already incorporated in system.

\subsection{Test Signals}

First step was to choose different test signals. All tests were made on 19 chosen test signals that are representing different types of PCCD OFDM ASK modulation techniques (Table 5). All these signals were carrying the same digital data (a digital data stream with 
Table 5

Configuration parameters of 19 pre-selected PCCD OFDM test signals

\begin{tabular}{ccccc}
\hline Signal & Number of carriers & First carrier $(\mathrm{Hz})$ & Symbol size & Bits per second \\
\hline 1 & 96 & 437.5 & 512 & 1500 \\
2 & 64 & 437.5 & 512 & 1000 \\
3 & 32 & 437.5 & 256 & 1000 \\
4 & 96 & 437.5 & 1024 & 750 \\
5 & 16 & 500 & 128 & 1000 \\
6 & 24 & 437.5 & 256 & 750 \\
7 & 24 & 2031.25 & 256 & 500 \\
8 & 32 & 3250 & 256 & 500 \\
9 & 32 & 437.5 & 512 & 500 \\
10 & 16 & 1625 & 256 & 500 \\
11 & 16 & 437.5 & 256 & 500 \\
12 & 8 & 500 & 128 & 500 \\
13 & 8 & 1625 & 128 & 500 \\
14 & 16 & 1625 & 512 & 250 \\
15 & 8 & 1625 & 256 & 250 \\
16 & 16 & 562.5 & 512 & 250 \\
17 & 8 & 562.5 & 256 & 250 \\
18 & 8 & 437.5 & 256 & 250 \\
19 & 16 & 437.5 & 512 & 250 \\
\hline
\end{tabular}

3200 bits) and the only difference between them was in number of carriers, first carrier frequency, symbol size in samples and bit rate that is consequence of three previous parameters.

\subsection{Offline Simulations}

Second step of verification was to make a program code for offline simulations of transmitting PCCD-OFDM-ASK through 3 different GSM coders. ETSI (European Telecommunications Standards Institute) suggested standard for all three GSM coders and the records-documentation is publicly available. Because of that, program code implementation was carried out and implemented into the MATLAB program environment for better understanding of working each component separately in the each GSM coder on signal level. ETSI has prescribed 16 test vectors, both input and output signals, used to check GSM coder implementation suitability.

All test signals were sent through each GSM coder separately for the purpose of analyzing influences and comparing and verifying results that were obtained with online simulations. 


\subsection{Online Simulations}

Third step of verification was to make online simulations of influences, caused by all three GSM coders. Online simulations were made on a professional equipment of Rohde\&Schwarz; unit "Universal Radio Communication Tester R\&S CMU 200”. This device has a RF part for communicating with mobile phones and it is capable to simulate all communication layers from RF part to higher layers like GPRS, SMS, etc. This device simulates a GSM base station where real mobile transaction is in place.

All 19 test signals were sent through each GSM coder separately and the result signals were later analyzed.

The results have shown that offline simulations are very much like online simulations. Table 6 and diagram depicted in Fig. 17 are presenting BER for each test signal separately on three different GSM coders. Based on these results, we have come to conclusion that the most appropriate combination of parameters for robust transmission are 2 types:

- 8 carriers, 256 samples per symbol, start carrier at frequency $437.5 \mathrm{~Hz}$ at $250 \mathrm{bps}$ (Signal 18),

- 16 carriers, 512 samples per symbol, start carrier at frequency $437.5 \mathrm{~Hz}$ at $250 \mathrm{bps}$ (Signal 19).

Table 6

BER size for different PCCD-OFDM-ASK modulation parameters in three different GSM coders

\begin{tabular}{cccc}
\hline Signal & $\begin{array}{c}\text { GSM-EFR } \\
\text { BER(\%) }\end{array}$ & $\begin{array}{c}\text { GSM-FR } \\
\text { BER(\%) }\end{array}$ & $\begin{array}{c}\text { GSM-HR } \\
\text { BER(\%) }\end{array}$ \\
\hline 1 & 5.53 & 22.75 & 34.16 \\
2 & 2.34 & 12.88 & 32.41 \\
3 & 2.06 & 13.00 & 28.75 \\
4 & 0.22 & 6.34 & 27.09 \\
5 & 2.88 & 13.03 & 26.22 \\
6 & 0.31 & 6.16 & 24.81 \\
7 & 0.16 & 33.34 & 21.47 \\
8 & 0.00 & 24.88 & 18.06 \\
9 & 0.00 & 0.75 & 17.5 \\
10 & 0.03 & 20.00 & 15.38 \\
11 & 0.00 & 0.78 & 15.22 \\
12 & 0.00 & 0.56 & 13.66 \\
13 & 0.00 & 17.19 & 10.53 \\
14 & 0.00 & 9.13 & 6.16 \\
15 & 0.00 & 6.09 & 5.06 \\
16 & 0.00 & 0.03 & 4.53 \\
17 & 0.00 & 0.00 & 4.22 \\
18 & 0.00 & 0.00 & 3.13 \\
19 & 0.00 & 0.00 &
\end{tabular}




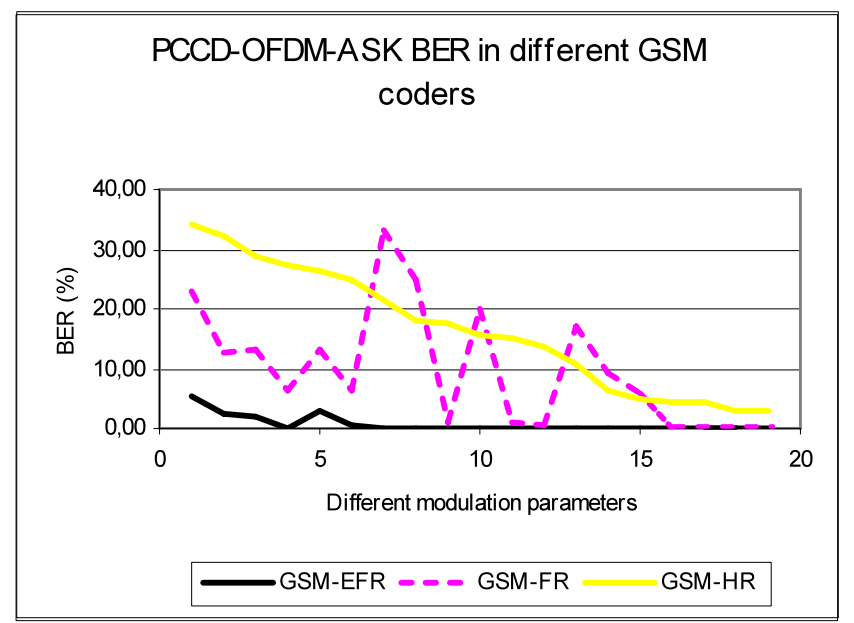

Fig. 17. BER size for different PCCD-OFDM-ASK modulation parameters in three different GSM coders.

\subsection{Real Environment Results}

Fourth step of verification was performed on real mobile transaction system applying a comparison to existing modulation techniques (ASK and FSK).

Existing modulations techniques do not have any special added signals or any other well-known technique for the purpose of detecting the start and end of the frame regardless to current BER values. Because of this no exact comparison on BER level can be made to show how better the new presented PCCD-OFDM-ASK technique is. Presented technique was mainly developed to improve transaction robustness. Transaction success-rate is sufficient information to say that we have improved communication between terminal and center. Above all, further improvements on PCCD-OFDM-ASK or any new modulation technique in the future can be directly compared on BER level. As we have measured these data and presented it above.

Transaction success on GSM-EFR and GSM-FR coder are not presented since the transaction success for all three modulation techniques is almost $100 \%$. On the contrary, tests on GSM-HR coder are showing great differences and the two diagrams in Fig. 18 are presenting them. All modulation techniques worked without any FEC techniques for better compare, although normally real system always work with different FEC techniques.

\section{Conclusion}

In this article a novel digital modulation-demodulation technique called PCCD-OFDMASK was presented. The proposed modulation technique is implemented in the commercially used mobile transaction system. The main purpose of the PCCD-OFDM-ASK is the transmission of the modulated digital data over the GSM or CDMA speech channel. The modulation procedure was specially developed to achieve very robust and reliable data transmission at bit rates up to $500 \mathrm{bps}$. 

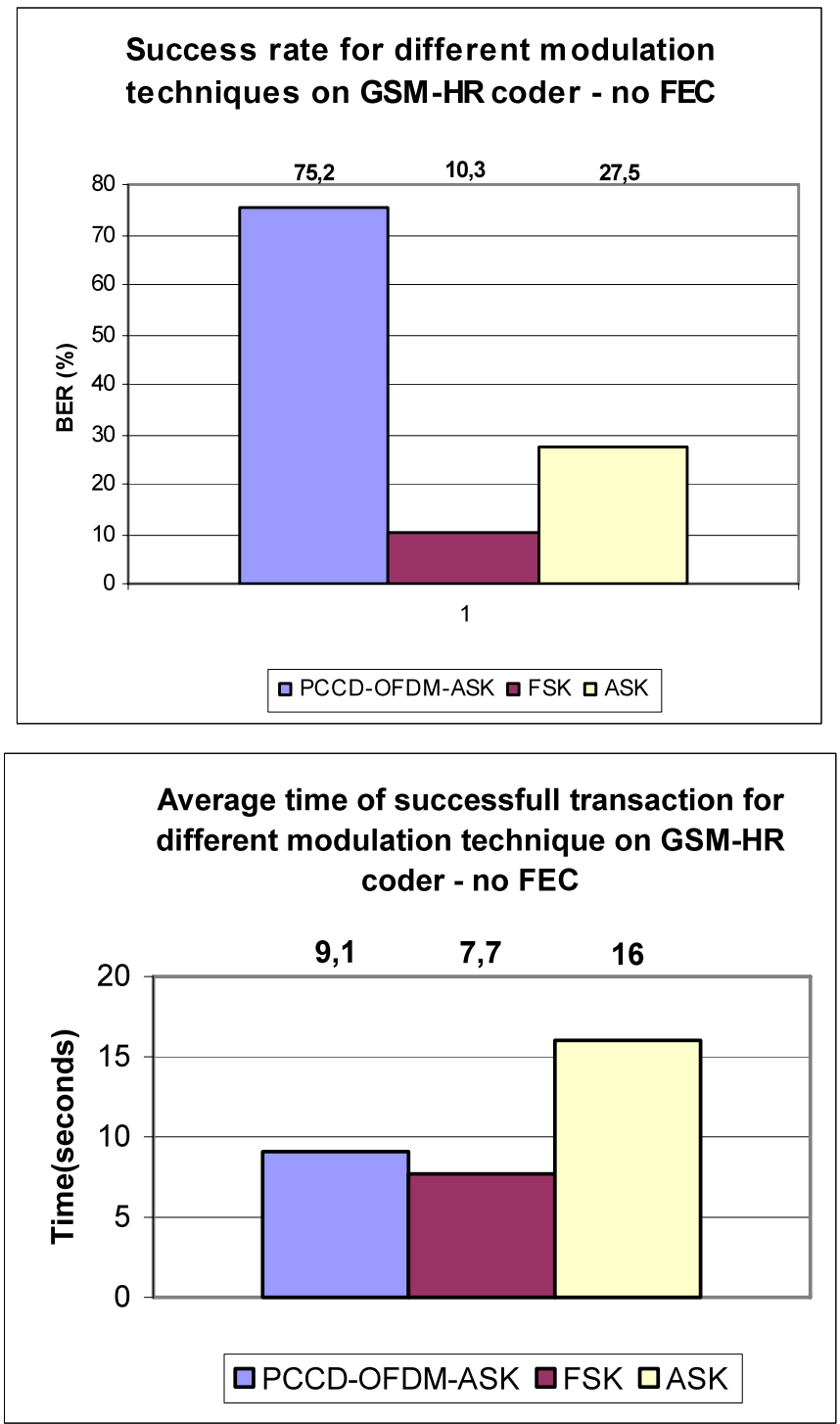

Fig. 18 (top and bottom diagrams). Comparison between different modulation techniques in real system on GSM-HR coder; without any forward error correction technique.

The modulator segments the input data stream into packets of 8 or 16 bits. The number of bits in each packet determines the number of orthogonal carriers. In contrast to classical OFDM, the lookup table is applied instead of IFFT to produce the samples of orthogonal carriers. The ASK principle is used to modulate the digital data on carriers: bit value ' 1 ' corresponds to enabled carrier, whereas bit value ' 0 ' means that the corresponding carrier will not exist in the particular symbol. Furthermore, the phase continuity is preserved between consecutive symbols: if particular bit does not change during two consecutive 
symbols, the phase of the corresponding carrier will be continuous. Otherwise, the rootraised signal shaping is applied with respect to context dependency principle. In order to achieve good demodulation robustness of the transmitted signal, a special adaptive magnitude threshold measuring procedure is integrated. This procedure estimates the frequency characteristic of the transmission channel several times during data transmission. In order to achieve precise symbol synchronization between the modulator and FFT-based demodulator, a special double-chirp synchronization signal was developed. Furthermore, the on-line energy normalization procedure has also been developed and integrated.

All offline and online data transmission simulations/experiments performed in the controlled laboratory environments have shown that PCCD-OFDM-ASK results are much better then those with the classic ASK and FSK based approaches. Transactions success rate over GSM-EFR, GSM-FR and GSM-HR speech coder is over 99\%, what is similar to the other technologies that were mentioned in the introduction section. Real system off course uses FEC techniques for improving data transmissions and because of that Figs. 18 and 19 are not mirroring real results that are happening on the field over the world. Results of new proposed modulation, where system is used, are much better toward old approached and this fact gives strong guideline for PCCD-OFDM based modulations. Because of that, further studies and research were made with PCCD-OFDM and first test results are showing new improvement and promising results. The already implemented original PCCD-OFDM-ASK digital data modulation principle will soon be transformed to even more robust novel PCCD-OFDM-FSK principle. This change will further improve the CDMA results that were slightly worst than GSM and affect of surround noise is reduced as well.

\section{References}

Batra, A., et al. (2004). Multi-band OFDM Physical Layer Proposal for IEEE 802.15 Task Group 3a. In IEEE document P802.15-04/0493r1, Vol. 9. Texas Instruments.

Bingham, J.A.C. (2000). ADSL, VDSL, and Multicarrier Modulation. John Wiley and Sons.

Chow, J.S., J.C. Tu and J.M. Cioffi (1991). A discrete multitone transceiver system for HDSL applications. IEEE J. Select. Areas Commun., 8, 895-908.

Davis, J.A., and J. Jedwab (1997). Peak-to-mean power control and error correction for OFDM transmission using Golay sequences and Reed-Muller codes. Electronic Letters, 33, 267-268.

Eneroth, P. (2001). Stereophonic acoustic echo cancellation. PhD dissertation, Lund University.

ETSI EN 300730 v7.0.1. (2000). Digital cellular telecommunications system-voice activity detector for enhanced full rate speech traffic channels (GSM 06.82). ETSI standard documentation.

GSM 06.10 Full Rate (FR) Vocoder (1996). Regular pulse excitation - long term prediction linear predictive coder (RPE-LTP). http: / / www . etsi. com

GSM 06.60 Enhanced Full Rate (EFR) Vocoder (1997). Algebraic-Code-Excited Linear Predictive (ACELP). http: / / www.etsi.com

GSM 06.20 Half Rate (HR) Vocoder (1998). Vector-Sum Excited Linear Prediction (VSELP). http: / /www.etsi.com

Gurprakash, S., and A. Arokiaswami (2003). OFDM modulation study for a radio-over-fiber system for wireless LAN (IEEE 802.11a). In Proc. ICICS-PCM 2003, Singapore.

Hanzo, L., F.C.A. Somerville and J.P. Woodard (1999). Voice Compression and Communications. IEEE Press - John Wiley and Sons.

Huang, H., S. Venkatesan, A. Kogiantis and N. Sharma (2003). Increasing the peak data rate of 3 G downlink packet data systems using multiple antennas. In Proc. Vehicular Technology Conference, Vol. 3. pp. 448. 
Ibars, C., and Y. Bar-Ness (2001). Comparing the performance of coded multiuser OFDM and coded MCCDMA over fading channels. In Proc. IEEE GLOBECOM Conference, Vol. 2. pp. 881-885.

Larsson, E.G., G. Liu, J. Li and G.B. Giannakis (2001). Joint symbol timing and channel estimation for OFDM based WLANs. In IEEE Communications Letters, Vol. 5. pp. 325-327.

Lawrey, E.P. (2001). Adaptive Techniques for Multiuser OFDM. PhD dissertation, James Cook University.

Lehtonen, K. (2004). Digital Signal Processing and Filtering - GSM Codec. Helsinki University of Technology.

Lipeika, A., and J. Lipeikiene (2008). On the use of the formant features in the dynamic time warping based recognition of isolated words. Informatica, 19(2), 213-226.

Nikolic, J., and Z. Peric (2008). Lloyd-Max's algorithm implementation in speech coding algorithm based on forward adaptive technique. Informatica, 19(2), 255-270.

Schmidl, T.M. et al. (1997). Robust frequency and timing synchronization for OFDM. IEEE Transactions on Communications, 45, 1613-1621.

Schmidt, M., and F. Jondral (2003). Ultra wideband transmission based on MCCDMA. In Proc. IEEE GLOBECOM Conference, Vol. 2. pp. 749-753.

Scourias, J. (1995). Overview of the global system for mobile communications. http://www.shoshin.uwaterloo.ca/ j.scouria/GSM/gsmreport.html, accessed 4 August 2008.

Sun, Y. (2001). Bandwidth-efficient wireless OFDM. IEEE Journal on Selected Areas in Communications, 19(11), 124.

Ultra M-Pay Patent 1 and 2 (2002). WO 02/33669, WO 03/088165.

Wiegandt, D.A. et al. (2003). High-throughput, high-performance OFDM via pseudo-orthogonal carrier interferometry spreading codes. IEEE Trans. Commun., 51(7), 1123-1134.

Xiong, F. (2006). Digital Modulation Techniques. Artech House Publishers.

Zhengdao, W., and G.B. Giannakis (2000). Wireless multicarrier communications. IEEE Signal Processing Magazine, 5, 1220 .

Z. Mezgec received his BSc degree in electrical engineering in 2004 and $\mathrm{PhD}$ in adaptive communications in 2008 both at University of Maribor, Slovenia. He started his research work in 2004 and since 2008 he has been working as chief of embedded system development at Ultra d.o.o. Research Center, Maribor, Slovenia. He also works since 2004 as young researcher in Robust Control Laboratory, Faculty of Electrical Engineering.

A. Chowdhury received his MSc degree in electrical engineering in 1997 and $\mathrm{PhD}$ in robust control in 2001 both at University of Maribor, Slovenia. Since 2005 he is head of Research Center at Ultra, M-Pay group, and beside this he is still working at University of Maribor, Faculty of Electrical Engineering and Computer Science, Slovenia.

R. Svečko received his MSc degree in electrical engineering in 1984 and $\mathrm{PhD}$ in control in 1989 both at University of Maribor, Slovenia. He works as a associates professor and researcher at University of Maribor, Faculty of Electrical Engineering and Computer Science, Slovenia.

B. Kotnik obtained his BSc degree in electrical engineering in 2000 and $\mathrm{PhD}$ in automatic speech recognition in 2004 both at University of Maribor, Slovenia. He is a developer and researcher in the fields of digital signal processing, digital modulation and demodulation algorithms, and statistical methods for data classification at Ultra Research Center, M-Pay group, Maribor, Slovenia. 


\title{
PCCD-OFDM-ASK robustinio duomenu perdavimo per GSM kalbos kanala igyvendinimas
}

\author{
Zdenko MEZGEC, Amor CHOWDHURY, Rajko SVEČKO, Bojan KOTNIK
}

Straipsnyje pateikiama nauja duomenu moduliacijos schema PCCD-OFDM-ASK: tolydinès fazès priklausomas nuo konteksto ortogonalių dažnių dalinimo maišymo poslinkio suderinimas. Pasiūlyta moduliacija yra sèkmingai pritaikyta mobilioje mokejimo sistemoje. Ji yra naudojama perduoti skaitmeninius duomenis mobilios ryšio sistemos GSM arba CDMA kalbos kanalu. Pagrindiniai pasiūlytos moduliacijos schemos elementai yra: tiksli signalo sinchronizacija tarp moduliatoriaus ir demoduliatoriaus, nepriklausomas nuo signalo energijos veikimas, perdavimo kanalo dažniniu charakteristiku adaptavimas realiame laike ir valdoma dažniu juosta, užtikrinanti pilnai dvipusị ryši GSM garso kanalu. 